Supporting Information for:

\title{
Developing Unique Non-Target High Resolution Mass Spectrometry Signatures to Track Contaminant Sources in Urban Waters
}

\author{
Bowen $\mathrm{Du}^{1 *}$, Zhenyu Tiann ${ }^{2,3}$, Katherine T. Peter ${ }^{4}$, Edward P. Kolodziejej,3,5, Charles S. Wong ${ }^{1}$ \\ ${ }^{1}$ Southern California Coastal Water Research Project Authority, Costa Mesa, CA 92626, USA \\ ${ }^{2}$ Interdisciplinary Arts and Sciences, University of Washington Tacoma, Tacoma, WA 98421, \\ USA \\ ${ }^{3}$ Center for Urban Waters, Tacoma, WA 98421, USA \\ ${ }^{4}$ National Institute of Standards and Technology, Charleston, SC 29412, USA \\ ${ }^{5}$ Department of Civil and Environmental Engineering, University of Washington, Seattle, WA \\ 98195, USA
}

October 9, 2020

11 pages, 8 tables, and 4 figures

Revised for submission to:

Environmental Science \& Technology Letters

*Corresponding author contact information

bowend@sccwrp.org 
1 Figure S1. Hierarchical cluster analysis of all water samples

2 Figure S2. Peak area abundances of select identified compounds.

3 Figure S3. Venn diagrams of non-target compounds in distinct wastewater types and road runoff 4 samples from different metro areas.

5 Figure S4. Normalized abundance analysis of the roadway runoff signature across metro areas.

7 Table S1. Sampling location information.

8 Table S2. Internal standards.

9 Table S3. Profinder data extraction and alignment settings.

10 Table S4. Wastewater and roadway runoff source signature feature lists (see Excel file).

11 Table S5. Identified compounds within the top 25 abundance detections for wastewater.

12 Table S6. Identified compounds within the top 25 abundance detections for roadway runoff.

13 Table S7. All identified compounds within the wastewater source signature (see Excel file).

14 Table S8. All identified compounds within the roadway runoff source signature (see Excel file).

17 Chemicals: Acetic acid ( $>99.7 \%$ ) and ammonium acetate (HPLC grade, $97.8 \%$ ) were purchased from VWR Scientific (Radnor, PA, USA). Methanol (MeOH: OPTIMA® grade) were obtained from Fisher Scientific (Fair Lawn, NJ, USA). A water purification system (Thermo Barnstead Nanopure Diamond UV, Dubuque, IA, USA) provided $18 \mathrm{M} \Omega$-cm water. 

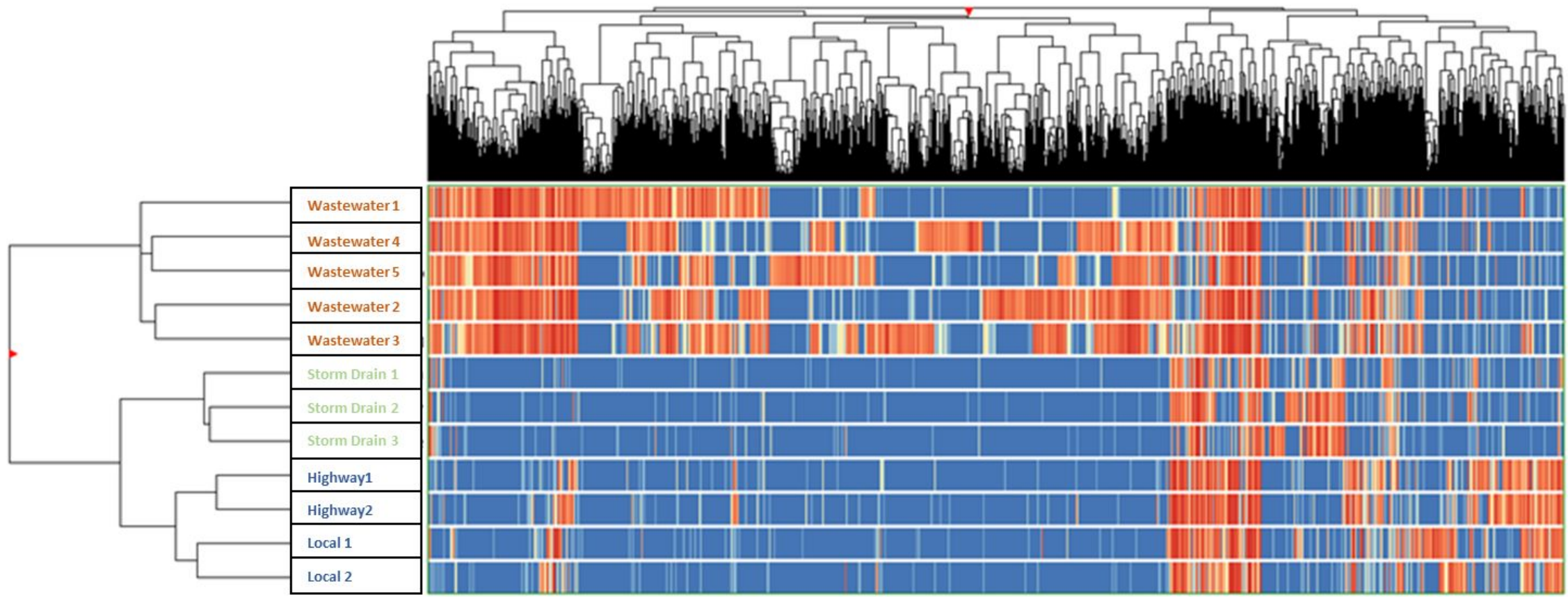

Figure S1. Hierarchical cluster analysis of all water samples, including wastewaters (red text), storm drain baseflow (blue text), and roadway runoff (green text), collected in Southern California. Each row represents an average of $3-5$ replicate samples, each vertical line represents an individual compound, and the color of each bar represents peak area (absent = blue; yellow, light red, and dark red with increasing peak area). 


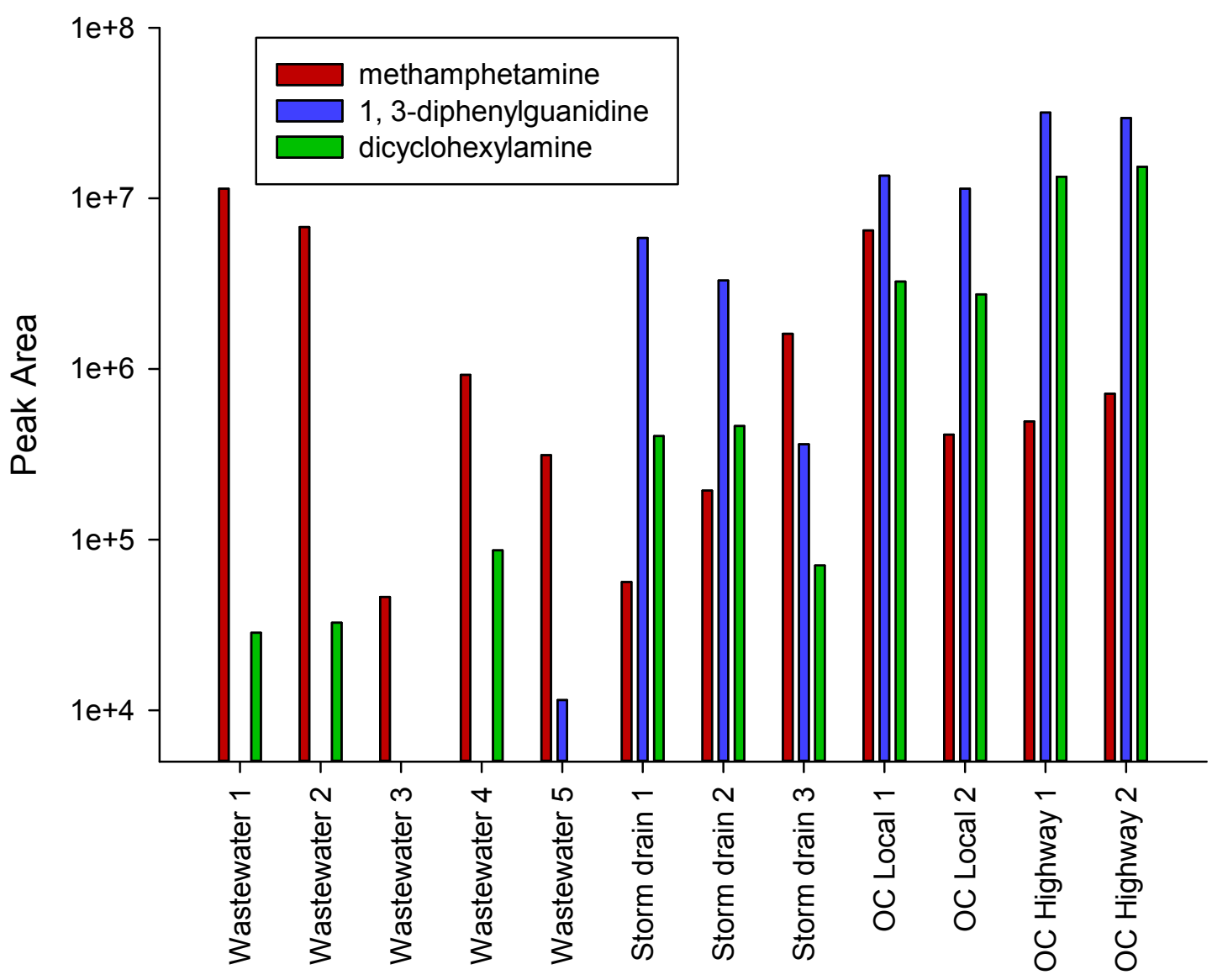

Figure S2. Peak abundances of methamphetamine, 1,3-diphenylguanidine, and dicyclohexylamine in waters in Southern California. Methamphetamine was detected in all samples. 1,3-diphenylguanidine was detected in all roadway runoff and storm drain samples, and in $20 \%$ of wastewater samples. Dicyclohexylamine was detected in all roadway runoff and storm drain samples, and in $60 \%$ of wastewater samples. OC $=$ Orange County. 

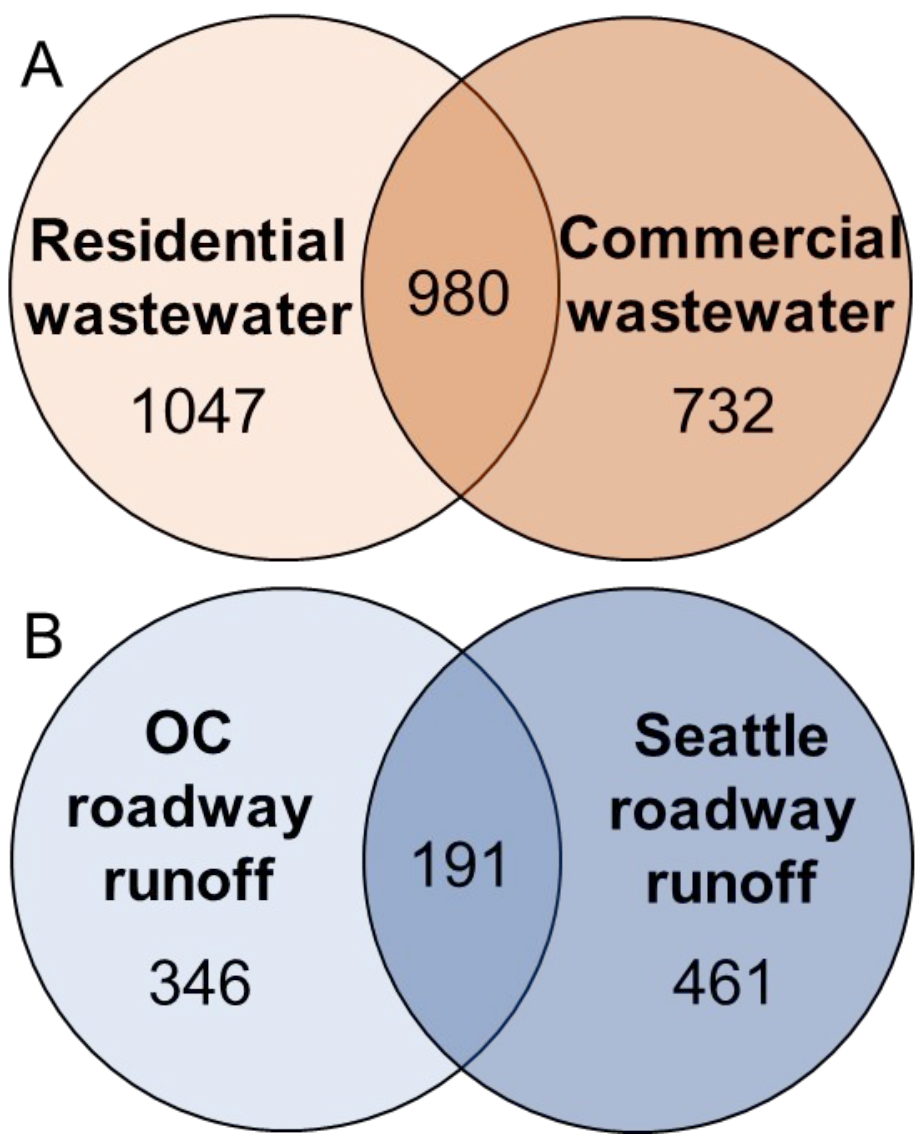

40

41 Figure S3. Venn diagram showing the overlap of compounds detected in (A) residential

42 (compounds in 2 of 2 samples) and commercial wastewater (compounds in 3 of 3 samples) and

43 (B) roadway runoff from Orange County, California (compounds in 4 of 4 samples) and Seattle,

44 Washington (compounds in 2 of 2 samples). 


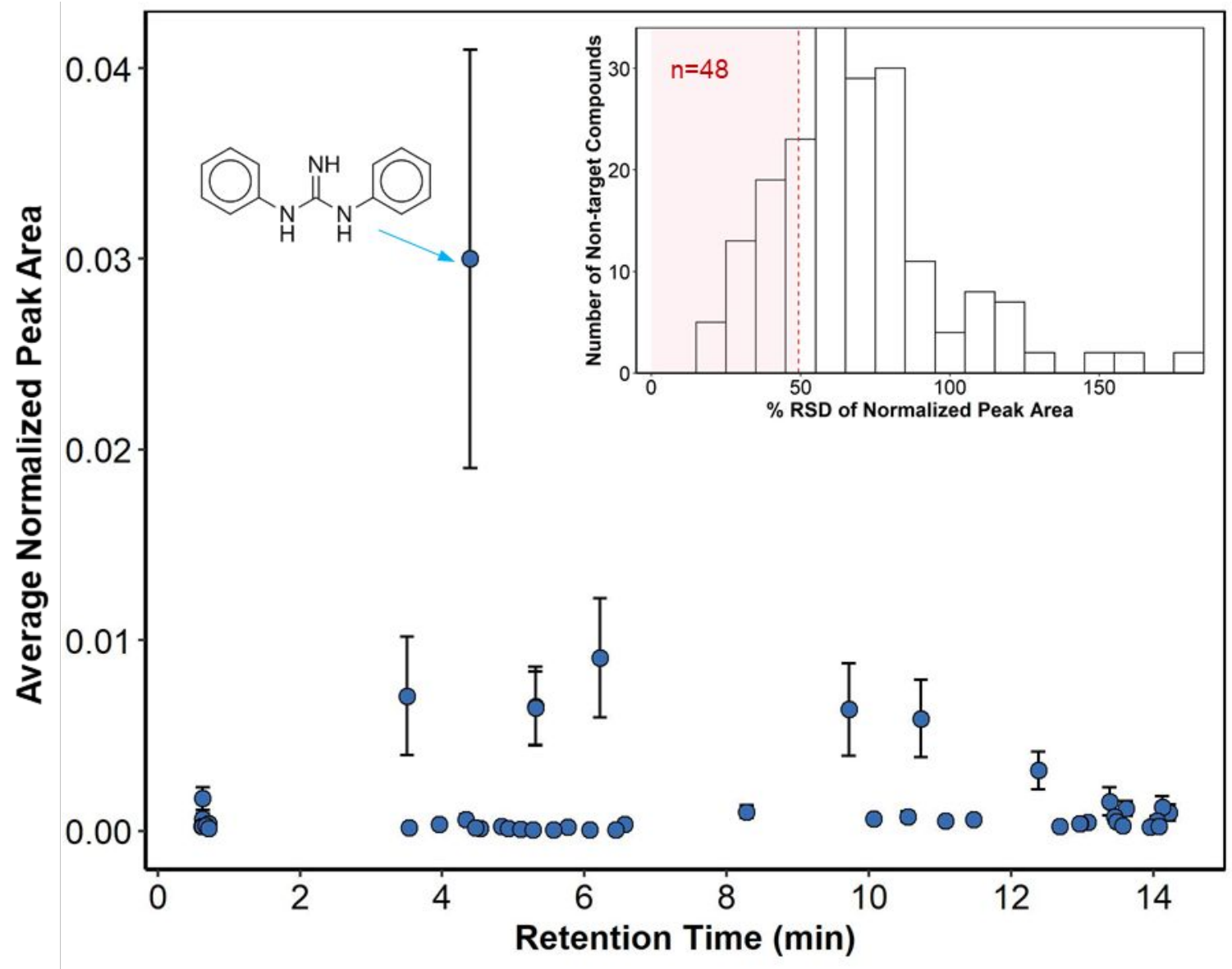

46 Figure S4. Average normalized peak area plotted against retention time for 48 compounds from 47 the roadway runoff chemical signature. The 48 compounds shown are those highlighted in pink 48 in the inset histogram, and were selected from the 191 signature compounds that were detected in 49 all six roadway runoff samples (all 191 are included in the histogram), based on relatively low 50 variability in their abundance pattern across the six samples. The variability threshold was set to 51 the upper end of the first quartile of the RSD distribution (RSD $<49 \%$ ). The structure of 1,352 diphenylguanidine is shown next to the corresponding data point. 
Table S1. Sampling locations.

\begin{tabular}{|c|c|c|c|c|c|}
\hline Name & Type & Latitude & Longitude & $\begin{array}{l}\text { Collection } \\
\text { dates }\end{array}$ & Comments \\
\hline Wastewater 1 & $\begin{array}{l}\text { Wastewater } \\
\text { influent }\end{array}$ & 32.835803 & -117.136934 & $01 / 24 / 2019$ & Commercial \\
\hline Wastewater 2 & $\begin{array}{l}\text { Wastewater } \\
\text { influent }\end{array}$ & 32.797496 & -117.160141 & $01 / 24 / 2019$ & $57 \%$ commercial $^{*}$ \\
\hline Wastewater 3 & $\begin{array}{l}\text { Wastewater } \\
\text { influent }\end{array}$ & 32.820917 & -117.155108 & $01 / 24 / 2019$ & Commercial \\
\hline Wastewater 4 & $\begin{array}{l}\text { Wastewater } \\
\text { influent }\end{array}$ & 32.839912 & -117.230352 & $01 / 24 / 2019$ & Residential \\
\hline Wastewater 5 & $\begin{array}{l}\text { Wastewater } \\
\text { influent }\end{array}$ & 32.834497 & -117.193694 & $01 / 24 / 2019$ & $79 \%$ residential $^{*}$ \\
\hline Storm Drain 1 & Baseflow & 32.800170 & -117.113430 & $05 / 08 / 2019$ & \\
\hline Storm Drain 2 & Baseflow & 32.834720 & -117.124200 & 05/08/2019 & \\
\hline Storm Drain 3 & Baseflow & 32.780280 & -117.051600 & $05 / 08 / 2019$ & \\
\hline $\begin{array}{l}\text { Orange County } \\
\text { Highway } 1\end{array}$ & $\begin{array}{l}\text { Roadway } \\
\text { runoff }\end{array}$ & 33.715876 & -117.789056 & $05 / 16 / 2019$ & \\
\hline $\begin{array}{l}\text { Orange County } \\
\text { Highway } 2\end{array}$ & $\begin{array}{l}\text { Roadway } \\
\text { runoff }\end{array}$ & 33.705973 & -117.854846 & 05/16/2019 & \\
\hline $\begin{array}{l}\text { Orange County } \\
\text { Local } 1\end{array}$ & $\begin{array}{l}\text { Roadway } \\
\text { runoff }\end{array}$ & 33.825453 & -117.851094 & $05 / 16 / 2019$ & \\
\hline $\begin{array}{l}\text { Orange County } \\
\text { Local } 2\end{array}$ & $\begin{array}{l}\text { Roadway } \\
\text { runoff }\end{array}$ & 33.826637 & -117.853049 & $05 / 16 / 2019$ & \\
\hline Seattle Highway 1 & $\begin{array}{l}\text { Roadway } \\
\text { runoff }\end{array}$ & 47.468111 & -122.306410 & $04 / 11 / 2019$ & \\
\hline Seattle Highway 2 & $\begin{array}{l}\text { Roadway } \\
\text { runoff }\end{array}$ & 47.643956 & -122.306858 & $12 / 20 / 2018$ & \\
\hline
\end{tabular}

${ }^{*}$ Wastewater type is based on percent of land use in the drainage area. 
58 Table S2. List of internal standards used for verifying instrumental performance.

\begin{tabular}{lcccc}
\hline Compound & Vendor & Formula & CAS No. & $\begin{array}{c}\text { Final } \\
\text { Conc. } \\
\text { (ng/mL) }\end{array}$ \\
\hline Carbamazepine-d $\mathrm{d}_{10}$ & Cerilliant & $\mathrm{C}_{15} \mathrm{H}_{2} \mathrm{D}_{10} \mathrm{~N}_{2} \mathrm{O}$ & $132183-78-9$ & 25 \\
Caffeine-13 $\mathrm{C}$ & Cerilliant & ${ }^{13} \mathrm{C}_{3} \mathrm{C}_{5} \mathrm{H}_{10} \mathrm{~N}_{4} \mathrm{O}_{2}$ & $78072-66-9$ & 50 \\
Cotinine- $\mathrm{d}_{3}$ & Cerilliant & $\mathrm{C}_{10} \mathrm{H}_{9} \mathrm{D}_{3} \mathrm{~N}_{2} \mathrm{O}$ & $110952-70-0$ & 100 \\
Sulfamethoxazole- $\mathrm{d}_{4}$ & CDN Isotopes & $\mathrm{C}_{10} \mathrm{H}_{7} \mathrm{D}_{4} \mathrm{~N}_{3} \mathrm{O}_{3} \mathrm{~S}$ & $1020719-86-1$ & 100 \\
Sulfadimethoxine- $\mathrm{d}_{6}$ & Sigma Aldrich & $\mathrm{C}_{12} \mathrm{D}_{6} \mathrm{H}_{8} \mathrm{~N}_{4} \mathrm{O}_{4} \mathrm{~S}$ & $73068-02-7$ & 100 \\
5-methyl-1H-benzotriazole- $\mathrm{d}_{6}$ & TRC Canada & $\mathrm{C}_{7} \mathrm{HD}_{6} \mathrm{~N}_{3}$ & $1246820-65-4$ & 100 \\
DEET-d & CDN Isotopes & $\mathrm{C}_{12} \mathrm{D}_{7} \mathrm{H}_{10} \mathrm{NO}$ & $1219799-37-7$ & 100 \\
Atrazine- $\mathrm{d}_{5}$ & CDN Isotopes & $\mathrm{C}_{8} \mathrm{H}_{5} \mathrm{D}_{5} \mathrm{ClN} \mathrm{N}_{5}$ & $163165-75-1$ & 100 \\
Prometon- $\mathrm{d}_{3}$ & CDN Isotopes & $\mathrm{C}_{10} \mathrm{H}_{16} \mathrm{D}_{3} \mathrm{~N}_{5} \mathrm{O}$ & $1219803-43-6$ & 100 \\
Metolachlor- $\mathrm{d}_{6}$ & CDN Isotopes & $\mathrm{C}_{15} \mathrm{H}_{16} \mathrm{D}_{6} \mathrm{ClNO}_{2}$ & $1219803-97-0$ & 100 \\
Bis(2-ethylhexyl)phthalate- $\mathrm{d}_{4}$ & CDN Isotopes & $\mathrm{C}_{24} \mathrm{H}_{34} \mathrm{D}_{4} \mathrm{O}_{4}$ & $93951-87-2$ & 100 \\
Nicotine- $\mathrm{d}_{3}$ & CDN Isotopes & $\mathrm{C}_{10} \mathrm{H}_{11} \mathrm{D}_{3} \mathrm{~N}_{2}$ & $69980-24-1$ & 500 \\
\hline
\end{tabular}


61 Table S3. Profinder data extraction and alignment settings.

\begin{tabular}{|c|c|c|}
\hline $\begin{array}{l}\text { Profinder Batch Recursive } \\
\text { Feature Extraction Wizard } \\
\text { Step }\end{array}$ & Category & Setting \\
\hline \multirow{3}{*}{$\begin{array}{l}\text { Molecular Feature Extraction } \\
\text { (MFE) - Extraction Parameters }\end{array}$} & Noise peak height & $\geq 400$ counts \\
\hline & Ion Species & $\begin{array}{l}+\mathrm{H},+\mathrm{Na},+\mathrm{NH} 4 \text { (salt- } \\
\text { dominated positive ions) }\end{array}$ \\
\hline & Isotope model & $\begin{array}{c}\text { Common organic } \\
\text { molecules }\end{array}$ \\
\hline \multirow{2}{*}{$\begin{array}{c}\text { Compound Binning and } \\
\text { Alignment }\end{array}$} & RT window & $\pm 0.3 \mathrm{~min}$ \\
\hline & Mass window & $\pm 20 \mathrm{ppm}$ \\
\hline \multirow{3}{*}{ MFE - Post-Processing Filters } & Absolute height & $\geq 5000$ counts \\
\hline & Score (MFE) & $\geq 70$ \\
\hline & Satisfy MFE conditions in & $\geq 2$ files \\
\hline \multirow[t]{2}{*}{$\begin{array}{l}\text { Find by Ion - Matching } \\
\text { Tolerances and Scoring }\end{array}$} & Scoring & $\begin{array}{c}\text { Mass score (100), isotope } \\
\text { abundance score }(60), \\
\text { isotope spacing score } \\
(50) \text {, retention time score } \\
(50)\end{array}$ \\
\hline & Do not match if score & $<30$ \\
\hline \multirow{2}{*}{$\begin{array}{l}\text { Find by Ion - EIC Peak } \\
\text { Integration and Filtering }\end{array}$} & Integration & Agile 2 \\
\hline & Peak height & $\geq 3000$ counts \\
\hline \multirow{3}{*}{$\begin{array}{c}\text { Find by Ion - Post-Processing } \\
\text { Filters }\end{array}$} & Absolute height & $\geq 1000$ counts \\
\hline & Score (Tgt) & $\geq 50$ \\
\hline & $\begin{array}{l}\text { Satisfy Find by Ion } \\
\text { conditions in }\end{array}$ & $\geq 2$ files \\
\hline
\end{tabular}

62 
Table S5. Top 25 abundance identifications shared by all wastewater samples (ranked by peak

\begin{tabular}{|c|c|c|c|c|c|c|c|c|}
\hline Name & CAS No. & Formula & Mass & $\mathbf{R T}^{\mathbf{a}}$ & Score $^{b}$ & $\begin{array}{l}\text { APA } \\
\text { (waste } \\
\text { water) }^{\mathrm{c}}\end{array}$ & $\begin{array}{l}\text { APA } \\
\text { (roadway } \\
\text { runoff) }\end{array}$ & $\begin{array}{l}\text { APA } \\
\text { (storm } \\
\text { drain) }\end{array}$ \\
\hline Decaethylene glycol & $5579-66-8$ & $\mathrm{C}_{20} \mathrm{H}_{42} \mathrm{O}_{11}$ & 458.2744 & 4.62 & 99.26 & $3.09 \mathrm{E}+07$ & $1.33 \mathrm{E}+07$ & $2.10 \mathrm{E}+05$ \\
\hline Nonaethylene glycol & $3386-18-3$ & $\mathrm{C}_{18} \mathrm{H}_{38} \mathrm{O}_{10}$ & 414.2477 & 4.45 & 99.51 & $2.66 \mathrm{E}+07$ & $1.41 \mathrm{E}+07$ & $2.64 \mathrm{E}+05$ \\
\hline Octaethylene glycol & $5117-19-1$ & $\mathrm{C}_{16} \mathrm{H}_{34} \mathrm{O}_{9}$ & 370.2211 & 4.24 & 99.69 & $2.47 \mathrm{E}+07$ & $1.73 \mathrm{E}+07$ & $3.48 \mathrm{E}+05$ \\
\hline Dodecaethylene glycol & $6790-09-6$ & $\mathrm{C}_{24} \mathrm{H}_{50} \mathrm{O}_{13}$ & 546.3279 & 4.88 & 98.39 & $2.34 \mathrm{E}+07$ & $5.67 \mathrm{E}+06$ & $5.06 \mathrm{E}+04$ \\
\hline Undecaethylene glycol & $6809-70-7$ & $\mathrm{C}_{22} \mathrm{H}_{46} \mathrm{O}_{12}$ & 502.3005 & 4.76 & 99.42 & $2.21 \mathrm{E}+07$ & $7.50 \mathrm{E}+06$ & $3.08 \mathrm{E}+04$ \\
\hline Tridecaethylene glycol & $17598-96-8$ & $\mathrm{C}_{26} \mathrm{H}_{54} \mathrm{O}_{14}$ & 590.3530 & 5.00 & 99.51 & $1.99 \mathrm{E}+07$ & $4.50 \mathrm{E}+06$ & $2.81 \mathrm{E}+04$ \\
\hline $\begin{array}{l}\text { Heptaethylene glycol } \\
\text { Tetradecaethylene }\end{array}$ & $5617-32-3$ & $\mathrm{C}_{14} \mathrm{H}_{30} \mathrm{O}_{8}$ & 326.1946 & 4.01 & 99.79 & $1.93 \mathrm{E}+07$ & $1.71 \mathrm{E}+07$ & $3.51 \mathrm{E}+05$ \\
\hline glycol & $67411-64-7$ & $\mathrm{C}_{28} \mathrm{H}_{58} \mathrm{O}_{15}$ & 634.3804 & 5.12 & 98.79 & $1.55 \mathrm{E}+07$ & $2.53 \mathrm{E}+06$ & $2.94 \mathrm{E}+04$ \\
\hline $\begin{array}{l}\text { Hexaethylene glycol } \\
\text { Pentadecaethylene }\end{array}$ & $2615-15-8$ & $\mathrm{C}_{12} \mathrm{H}_{26} \mathrm{O}_{7}$ & 282.1684 & 3.74 & 99.77 & $1.48 \mathrm{E}+07$ & $1.35 \mathrm{E}+07$ & $9.97 \mathrm{E}+05$ \\
\hline glycol & $28821-35-4$ & $\mathrm{C}_{30} \mathrm{H}_{62} \mathrm{O}_{16}$ & 678.4063 & 5.23 & 99.12 & $1.34 \mathrm{E}+07$ & $2.07 \mathrm{E}+06$ & $5.21 \mathrm{E}+06$ \\
\hline $\begin{array}{l}\text { Pentaethylene glycol } \\
\text { Nonadecaethylene }\end{array}$ & $4792-15-8$ & $\mathrm{C}_{10} \mathrm{H}_{22} \mathrm{O}_{6}$ & 238.1429 & 3.46 & 98.58 & $8.23 \mathrm{E}+06$ & $7.56 \mathrm{E}+06$ & $1.72 \mathrm{E}+06$ \\
\hline $\begin{array}{l}\text { glycol } \\
\text { Heptadecaethylene }\end{array}$ & & $\mathrm{C}_{38} \mathrm{H}_{78} \mathrm{O}_{20}$ & 854.5116 & 5.70 & 0914 & $5.99 \mathrm{E}+06$ & $1.64 \mathrm{E}+05$ & $7.46 \mathrm{E}+04$ \\
\hline $\begin{array}{l}\text { glycol } \\
\text { Heptacosaethylene }\end{array}$ & & $\mathrm{C}_{34} \mathrm{H}_{70} \mathrm{O}_{18}$ & 766.4583 & 5.46 & 99.13 & $4.98 \mathrm{E}+06$ & $3.13 \mathrm{E}+06$ & $1.94 \mathrm{E}+06$ \\
\hline $\begin{array}{l}\text { glycol } \\
\text { Octaethylene glycol }\end{array}$ & & $\mathrm{C}_{54} \mathrm{H}_{110} \mathrm{O}_{28}$ & 1206.728 & 6.71 & 93.97 & $4.91 \mathrm{E}+06$ & $\mathrm{ND}^{\mathrm{d}}$ & ND \\
\hline $\begin{array}{l}\text { monodecyl ether } \\
\text { Heptaethylene glycol }\end{array}$ & $24233-81-6$ & $\mathrm{C}_{26} \mathrm{H}_{54} \mathrm{O}_{9}$ & 510.3800 & 13.48 & 97.79 & $4.77 \mathrm{E}+06$ & $1.07 \mathrm{E}+06$ & $4.67 \mathrm{E}+06$ \\
\hline $\begin{array}{l}\text { monodecyl ether } \\
\text { Nonaethylene glycol }\end{array}$ & $39840-09-0$ & $\mathrm{C}_{24} \mathrm{H}_{50} \mathrm{O}_{8}$ & 466.3539 & 13.43 & 97.15 & $4.68 \mathrm{E}+06$ & $1.14 \mathrm{E}+06$ & $1.04 \mathrm{E}+04$ \\
\hline monodecyl ether & $6008-31-7$ & $\mathrm{C}_{28} \mathrm{H}_{58} \mathrm{O}_{10}$ & 554.4059 & 13.52 & 98.39 & $4.37 \mathrm{E}+06$ & $8.10 \mathrm{E}+05$ & ND \\
\hline $\begin{array}{l}\text { Methamphetamine } \\
\text { Decaethylene glycol }\end{array}$ & $537-46-2$ & $\mathrm{C}_{10} \mathrm{H}_{15} \mathrm{~N}$ & 149.1207 & 3.93 & 87.95 & $3.88 \mathrm{E}+06$ & $1.94 \mathrm{E}+06$ & $6.16 \mathrm{E}+05$ \\
\hline $\begin{array}{l}\text { monodecyl ether } \\
\text { Hexaethylene glycol }\end{array}$ & $18554-67-1$ & $\mathrm{C}_{30} \mathrm{H}_{62} \mathrm{O}_{11}$ & 598.4324 & 13.56 & 98.35 & $3.72 \mathrm{E}+06$ & $5.62 \mathrm{E}+05$ & ND \\
\hline & $5168-89-8$ & $\mathrm{C}_{22} \mathrm{H}_{46} \mathrm{O}_{7}$ & 422.3323 & 13.31 & 83.42 & $3.56 \mathrm{E}+06$ & $1.04 \mathrm{E}+06$ & $1.09 \mathrm{E}+04$ \\
\hline Tetraethylene glycol & $\begin{array}{l}112-60-7 \\
351342-08-\end{array}$ & $\mathrm{C}_{8} \mathrm{H}_{18} \mathrm{O}_{5}$ & 194.1167 & 3.03 & 98.36 & $3.37 \mathrm{E}+06$ & $5.35 \mathrm{E}+06$ & $2.37 \mathrm{E}+05$ \\
\hline Eicosaethylene glycol & 0 & $\mathrm{C}_{40} \mathrm{H}_{82} \mathrm{O}_{21}$ & 898.5416 & 5.82 & 96.11 & $3.29 \mathrm{E}+06$ & $8.30 \mathrm{E}+04$ & $5.84 \mathrm{E}+03$ \\
\hline \multirow{2}{*}{$\begin{array}{l}\text { Caffeine } \\
\text { Undecaethylene glycol } \\
\text { monodecyl ether }\end{array}$} & $58-08-2$ & $\mathrm{C}_{8} \mathrm{H}_{10} \mathrm{~N}_{4} \mathrm{O}_{2}$ & 194.0813 & 4.01 & 99.05 & $3.14 \mathrm{E}+06$ & $1.95 \mathrm{E}+05$ & $4.63 \mathrm{E}+04$ \\
\hline & & $\mathrm{C}_{32} \mathrm{H}_{66} \mathrm{O}_{12}$ & 642.4600 & 13.60 & 96.94 & $2.58 \mathrm{E}+06$ & $4.07 \mathrm{E}+05$ & ND \\
\hline Ranitidine & $66357-35-5$ & $\mathrm{C}_{13} \mathrm{H}_{22} \mathrm{~N}_{4} \mathrm{O}_{3} \mathrm{~S}$ & 314.1434 & 3.13 & 97.53 & $2.52 \mathrm{E}+06$ & ND & ND \\
\hline
\end{tabular}

a. $\mathrm{RT}=$ retention time $(\mathrm{min})$

b. Based on specified adducts, the software automatically searched the presence of the precursor ions of compounds and assigned a cumulative score to matches based on mass accuracy, isotopic spacing, and relative isotope abundance.

d. $\quad \mathrm{ND}=$ not detected 
Table S6. Top 25 abundance identifications shared by all roadway runoff samples (ranked by peak area).

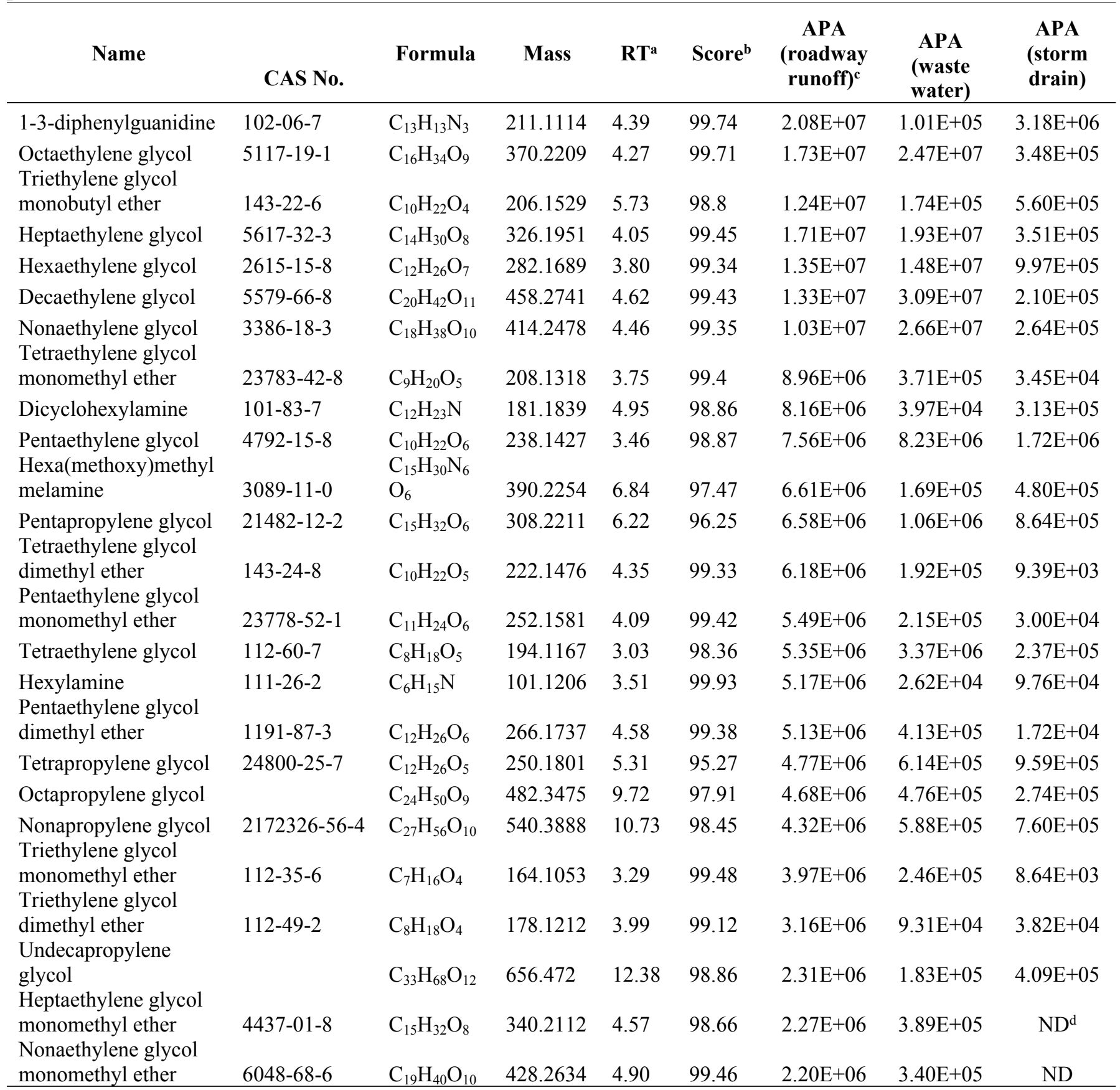
a. $\quad \mathrm{RT}=$ retention time $(\mathrm{min})$
b. Based on specified adducts, the software automatically searched the presence of the precursor ions of compounds and assigned a cumulative score to matches based on mass accuracy, isotopic spacing, and relative isotope abundance.
c. $\mathrm{APA}=$ average peak area
d. $\quad$ ND $=$ not detected 